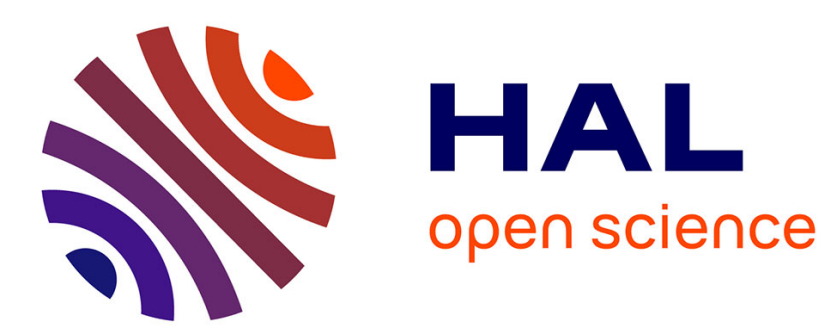

\title{
An introduction to simple sets
}

Nicolas Passat, Loïc Mazo

\section{- To cite this version:}

Nicolas Passat, Loïc Mazo. An introduction to simple sets. Pattern Recognition Letters, 2009, 30

(15), pp.1366-1377. 10.1016/j.patrec.2009.07.008 . hal-01694415

\section{HAL Id: hal-01694415 \\ https://hal.univ-reims.fr/hal-01694415}

Submitted on 26 Feb 2018

HAL is a multi-disciplinary open access archive for the deposit and dissemination of scientific research documents, whether they are published or not. The documents may come from teaching and research institutions in France or abroad, or from public or private research centers.
L'archive ouverte pluridisciplinaire HAL, est destinée au dépôt et à la diffusion de documents scientifiques de niveau recherche, publiés ou non, émanant des établissements d'enseignement et de recherche français ou étrangers, des laboratoires publics ou privés. 
Elsevier Editorial System(tm) for Pattern Recognition Letters Manuscript Draft

Manuscript Number: PATREC-D-08-00467R2

Title: An introduction to simple sets

Article Type: Regular Article

Keywords: Discrete topology; Homotopy type preservation; Simple sets; Cubical complexes; n-D spaces.

Corresponding Author: Dr. Nicolas Passat,

Corresponding Author's Institution: Strasbourg 1 University

First Author: Nicolas Passat

Order of Authors: Nicolas Passat; Loïc Mazo 


\section{Pattern Recognition Letters}

\section{Authorship Confirmation}

Please save a copy of this file, complete and upload as the "Confirmation of Authorship" file.

As corresponding author, I Nicolas Passat, hereby confirm on behalf of all authors that:

1. This manuscript, or a large part of it, has not been published, was not, and is not being submitted to any other journal. If presented at a conference, the conference is identified. If published in conference proceedings, the publication is identified below and substantial justification for re-publication must be presented.

2. All text and graphics, except for those marked with sources, are original works of the authors, and all necessary permissions for publication were secured prior to submission of the manuscript.

3. All authors each made a significant contribution to the research reported and have read and approved the submitted manuscript.

Date 06 August 2008

Previous conference presentation

Previous conference proceedings publication

Justification for re-publication 


\section{An introduction to simple sets PATREC-D-08-00467 Answers to reviewers}

July 12, 2009

\section{Reviewer 2}

The paper is now acceptable for publication, as the overall quality has been greatly increased by the answers given to the reviewers' comments. I just have two minor corrections and one request.

- Page 12: "preserves homotopy type" $\rightarrow$ "preserves the homotopy type".

Answer: This has been corrected.

- Page 13, remark 8:

The collapsing operation you define here corresponds to the very same operation, the collapsing, of (Kong 1997). The link between the latter and the deformation retraction is the purpose of Proposition 10 in Kong's paper (although deformation retraction is not mentioned).

Your sentence may suggest that Kong have defined the operation of deformation retraction, which is not correct. It is a classical notion in topology. Neither did he define collapsing. He used these tools of combinatorial topology to make a link between the definition of simple points on the one hand, and the classical notion of continuous deformation on the other hand. Kong himself indeed refers to Proposition 10 as a "well known fact" and to collapsing as "a standard tool of geometrical combinatorial topology".

Your remark 8 is certainly worth being done, as an answer to one of my comments on your first submitted paper, and it should also definitely refer to the work of Kong. But you should formulate it differently.

Answer: Remark 8 has been modified accordingly.

- Figure 23: The (l) is italic with no apparent reason.

Answer: Unfortunately, this seems to be a side effect of the elsart package (it does not happen when compiling in "article" mode instead of "elsart" ("elsart3").

A new reference has been added (Whitehead, 1938) to enable the modification of Remark 8, while the reference (Passat et al. 2009) has been updated. 


\title{
An introduction to simple sets
}

\author{
Nicolas Passat ${ }^{\text {a,* }}$, Loïc Mazo ${ }^{\text {a }}$ \\ ${ }^{a}$ Université de Strasbourg, LSIIT, UMR CNRS 7005, France
}

\begin{abstract}
Preserving topological properties of objects during thinning procedures is an important issue in the field of image analysis. In this context, we present an introductory study of the new notion of simple set which extends the classical notion of simple point. Similarly to simple points, simple sets have the property that the homotopy type of the object in which they lie is not changed when such sets are removed. Simple sets are studied in the framework of cubical complexes which enables, in particular, to model the topology in $\mathbb{Z}^{n}$. The main contributions of this article are: a justification of the study of simple sets (motivated by the limitations of simple points); a definition of simple sets and of a sub-family of them called minimal simple sets; the presentation of general properties of (minimal) simple sets in $n$-D spaces, and of more specific properties related to "small dimensions" (these properties being devoted to be further involved in studies of simple sets in 2, 3 and 4-D spaces).
\end{abstract}

Key words: Discrete topology, homotopy type preservation, simple sets, cubical complexes, $n$-D spaces.

\section{Introduction}

Topological properties are fundamental in many applications of image analysis, in particular in research fields where the retrieval and/or preservation of topology of real complex structures is required.

Topology-preserving operators, like homotopic skeletonisation, are used to transform an object while leaving unchanged its topological characteristics. In discrete grids $\left(\mathbb{Z}^{2}, \mathbb{Z}^{3}\right.$, or $\left.\mathbb{Z}^{4}\right)$, such transformations can be defined and efficiently implemented thanks to the notion of simple point (Kong and Rosenfeld (1989); Bertrand (1994); Couprie and Bertrand (2009)): intuitively, a point of an object is called simple if it can be deleted from this object without altering its topology. A typical

\footnotetext{
* Corresponding author: Nicolas Passat

(email: passat@unistra.fr, tel: (+33) 3688544 96, fax: (+33) 3688544 55).
} 
topology-preserving transformation based on simple points deletion, that we call guided homotopic thinning (Davies and Plummer (1981); Couprie et al. (2007)), may be described as follows. The input data consists of a set $X$ of points in the grid (called object), and a subset $K \subset X$ (called constraint set). Let $X_{0}=X$. At each iteration $i$, choose a simple point $x_{i} \in X_{i} \backslash K$ according to some criterion (e.g., a priority function) and set $X_{i+1}=X_{i} \backslash\left\{x_{i}\right\}$. Continue until reaching a step $n$ such that no simple point for $X_{n}$ remains in $X_{n} \backslash K$. We call the result of this process a homotopic skeleton of $X$ constrained by $K$. Notice that, since several points may have the same priority, there may exist several homotopic skeletons for a given pair $(X, K)$.

The most common example of priority function for the choice of $x_{i}$ is a distance map which associates to each point of $X$ its distance from the boundary of $X$. In this case, the points which are closest to the boundary are chosen first, resulting in a skeleton which is "centered" in the original object. In some particular applications, the priority function may be obtained through a grey-scale image, for example when the goal is to segment objects in this image while respecting topological constraints (Dokládal et al. (1999)). In the latter case, the order in which points are considered does not rely on geometrical properties, and may be affected by noise.

One drawback of thinning algorithms that work in the manner we have described is that the final set $X_{n}$ is not always minimal (Passat et al. (2005)). The problem here is that even though $X_{n}$ contains no simple point outside the constraint set $K$, it is still possible for $X_{n} \backslash K$ to include non-empty subsets $D$ which have the property that $X_{n}$ can be "deformed", in a sense that will be made precise in Definition 7, onto the smaller set $X_{n} \backslash D$ (so that $X_{n}$ is "homotopy equivalent" in a discrete sense to $X_{n} \backslash D$ ). A subset $D$ that has this property will be called a simple set (for $X_{n}$ ). An example of such a set is depicted in Fig. 1; if $X_{n}$ is the 3-D set shown in that figure, then the set $D \subset X_{n}$ (in light grey) is simple for $X_{n}$.

One way to address this problem would be to try to further reduce the set $X_{n}$ by finding and deleting some subset $D$ of $X_{n} \backslash K$ that is simple for $X_{n}$. To put this idea into practice, we need good ways of finding sets in $X_{n} \backslash K$ that are simple for $X_{n}$.

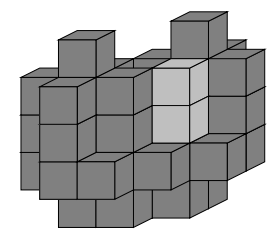

Fig. 1. A set $X_{n}$ composed of 32 points (considered in 26-adjacency, see also Figs. 3(a) and 4(a)), which does not contain any simple point, but which includes a subset $D$ (in light grey), whose removal from $X_{n}$ provides a set $X_{n} \backslash D$ "homotopy equivalent" in a discrete sense to $X_{n}$. In particular, it is possible to further reduce $X_{n} \backslash D$ to a single point by iterative removal of simple points.

We are, in particular, interested by simple sets which are minimal, in the sense that 
they do not strictly include any other simple set, since it is sufficient to detect such sets in order to carry on thinning. Also, we can hope that such minimal simple sets (i) have a specific structure which could make them easier to analyse, and (ii) are sufficient to deal with the whole problem of simple set removal.

The sequel of this article is organised as follows. In Section 2, we propose a discussion on "topological artifacts" which may appear in discrete images, especially during topology-preserving reduction procedures (generally based on simple points). This discussion leads to define the notion of simple set which provides a way to "break" some of these artifacts, and then enables to improve the efficiency of reduction procedures. In Section 3, the framework of cubical complexes is described. Indeed, we propose to study simple sets in this framework, from which we can retrieve the classical notions of digital topology in $\mathbb{Z}^{n}$, but which also enables to deal with more general categories of cubical objects. Section 4 presents the main notions of topology preservation in cubical spaces and formally introduces the definitions of simple sets and minimal simple sets. General properties of such sets (valid in any dimension) are proposed and proved in Section 5, while more specific ones, devoted to "low dimensions" are proposed and proved in Section 6. Discussions and perspectives regarding further works on simple sets are provided in Section 7.

\section{Why are simple sets useful?}

\subsection{Topological artifacts: the notion of lump}

Let us consider the guided homotopic thinning procedure described in Section 1. When performing such a procedure, the result is expected to fulfil a property of minimality. This is indeed the case since the result $X_{n}$ is minimal in the sense that it contains no simple point outside of $K$. However, we could formulate a stronger minimality requirement, which seems natural for this kind of transformation: informally, the result $X_{n}$ should not strictly include any subset $Y$ which is "topologically equivalent" to $X$, and such that $K \subseteq Y \subset X_{n}$. We say that a homotopic skeleton of $X$ constrained by $K$ is globally minimal if it fulfils this condition.

Now, a fundamental question arises: is any homotopic skeleton globally minimal? Let us illustrate this problem in dimensions 2 and 3 . In $\mathbb{Z}^{2}$, consider a full rectangle $X$ of any size, and the constraint set $K=\emptyset$. Obviously, this object $X$ is topologically equivalent to a single point, thus only homotopic skeletons which are singletons are globally minimal. Rosenfeld (1970) proved that any homotopic skeleton of $X$ is indeed reduced to a single point.

However, in dimensions $n \geq 3$, this property does not hold: if $X$ is $e$.g. a full $k \times k \times k$ cube $(k \geq 5)$, we may find a homotopic skeleton of $X$, with empty constraint set, 
which is not reduced to a single point (see Fig. 1). A classical counter-example is the Bing's house with two rooms (Bing (1964)), illustrated in Fig. 2. One can enter the lower room of the house by the chimney passing through the upper room, and vice versa. A discrete version $X_{1}$ of the Bing's house is displayed in Fig. 5(a). It can be seen that the Bing's house can be carved from a full cube by iterative removal of simple points. It can also be seen that $X_{1}$ contains no simple point: deleting any point from $X_{1}$ would create a "tunnel".

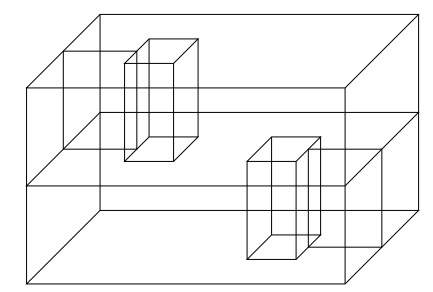

Fig. 2. A Bing's house with two rooms visualised as a 2-D surface in $\mathbb{R}^{3}$ (see text).

It could be argued that objects like Bing's houses are unlikely to appear while processing real (noisy) images, because of their complex shape and their size. However, Passat et al. (2007) found that there exists a large class of objects (of any topology) presenting similar properties, some of them being quite small (see Figs. 3 and 4). Such objects will be called lumps and can be defined, as follows, thanks to the notion of simple-equivalence.

Definition 1 Let $n \geq 1$. Let $X, X^{\prime} \subset \mathbb{Z}^{n}$. We say that $X$ and $X^{\prime}$ are simple-equivalent if there exists a sequence of sets $\left\langle X_{i}\right\rangle_{i=0}^{t}(t \geq 0)$ such that $X_{0}=X, X_{t}=X^{\prime}$, and for all $i \in[1, t]$, we have either:

(i) $X_{i}=X_{i-1} \backslash\left\{x_{i}\right\}$, where $x_{i} \in X_{i-1}$ is a simple point for $X_{i-1}$; or

(ii) $X_{i-1}=X_{i} \backslash\left\{x_{i}\right\}$, where $x_{i} \in X_{i}$ is a simple point for $X_{i}$.

Definition 2 Let $n \geq 1$. Let $X^{\prime} \subset X \subset \mathbb{Z}^{n}$ such that $X$ and $X^{\prime}$ are simple-equivalent. If $X$ does not contain any simple point outside $X^{\prime}$, then we say that $X$ is a lump relative to $X^{\prime}$, or simply a lump.

Note that the definition of lumps is proposed here in $\mathbb{Z}^{n}$. In the next sections, we will propose a definition of lumps in the framework of cubical complexes. Once introduced the basic notions on cubical complexes (Section 3), this new definition will generalise the current one.

Motivated by these considerations, mainly illustrated in $3-\mathrm{D}$, but still valid in $n$-D, two questions arise: is it possible to detect when a thinning procedure gets stuck on a lump, and then, is it possible to find a way towards a globally minimal homotopic skeleton? For performing the latter task, a solution consists in identifying a subset of $X$ which can be removed without changing topology: we will call such a subset a simple set. 


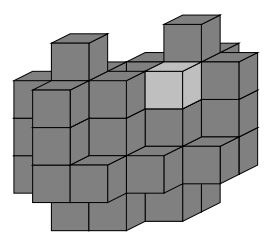

(a)

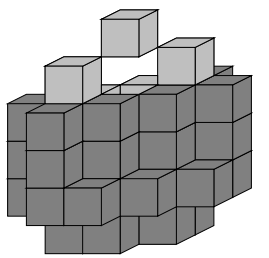

(b)

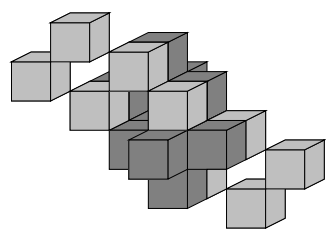

(c)

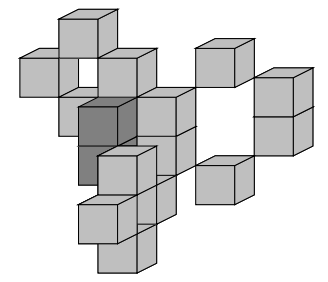

(d)

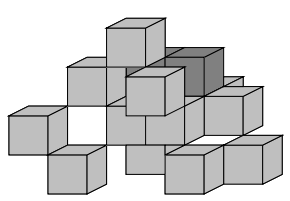

(e)

Fig. 3. Examples of 3-D lumps $L_{i}$ ((a-e): $L_{1}$ to $\left.L_{5}\right)$, considered in 26-adjacency. Topologically equivalent subsets are depicted in light grey. The set $L_{i}(i=1$ to 5 ) has $i-1$ tunnel(s).

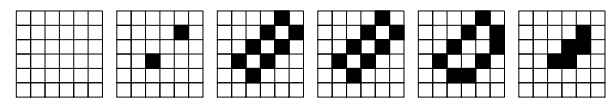

(a)

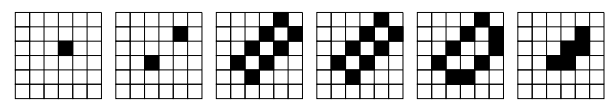

(b)

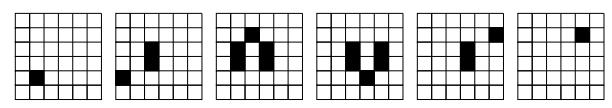

(c)

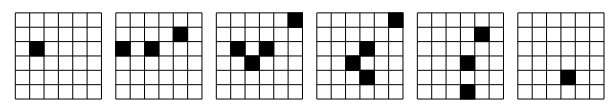

(d)

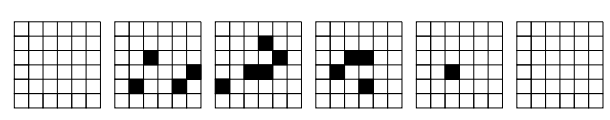

(e)

Fig. 4. 2-D visualisation of the successive planar slices of the lumps $L_{i}$ of Fig. 3. (a-e): $L_{1}$ to $L_{5}$ (in black). 


\subsection{Simple sets}

Intuitively, a simple set can be defined as a subset $Y$ of an object $X$ whose removal from $X$ "does not alter the topology of $X$ ". Following this intuitive and informal definition, the sets $Y_{i} \subset X_{i}$ (see Figs. 5-7) are simple for the set in which they lie (in particular, $X_{i}$ and $X_{i} \backslash Y_{i}$ are simple-equivalent).

However, among these sets, we may distinguish two categories: the sets $Y_{3}, Y_{4}$, and $Y_{5}$ (see Fig. 7) are different from the sets $Y_{1}$ and $Y_{2}$ (see Figs. 5 and 6) since the removal of the first ones can be obtained by monotonically reducing the object (in a "continuous-like" fashion which will be detailed in Definition 7), while the removal of the other ones is necessarily the result of a non-monotonic (i.e. reduction and growing) procedure: these two simple sets are defined as the result of the subtraction of the final and initial (simple-equivalent) objects.

From now on, we will only call simple set a set corresponding to the first category. This is justified by two arguments: $(i)$ such sets are defined from a monotonic transformation (with respect to the inclusion relation), which is consistent with the general use of topology-preserving operations, that generally consist of reduction or (dual) growing processes, and (ii) by definition such objects may be locally characterised (such local characterisations being generally impossible for the sets of the second category ${ }^{1}$ ), which provides a real interest for their involvement in the development of efficient topology-preserving procedures.

Let us now consider the simple sets $Y_{i}(i \in[3,5])$ of Fig. 7. The set $Y_{3}$ is obviously composed of two points $x$ and $y$ being both simple for $X_{3-5}$. The removal of $x$ (resp. $y$ ) from $X_{3-5}$ then does not alter its topology. Moreover, this is also true for the iterative (or parallel) removal of $x$ and $y$ from $X_{3-5}$. Such points, called P-simple points, have been fully described and characterised by Bertrand (1995). It is easy to observe that any set $Y$ composed of points being P-simple for a set $X$ can be removed from $X$ without altering its topology (such a removal corresponding to the successive removal of the points of $Y$ in any order, and then to a monotonic reduction of $X$ ), thus is a simple set for $X$.

The set $Y_{4}$ is composed of two points $x$ and $y$ such that $x$ is simple for $X_{3-5}$ while $y$ is simple for $X_{3-5} \backslash\{x\}$ but not for $X_{3-5}$. Consequently, the iterative removal of $x$ and $y$ (in the correct order) from $X_{3-5}$ does not alter its topology. Any set $Y$ composed, as $Y_{4}$, of such "successively" simple points for a set $X$ is then a simple set for $X$. This subfamily of simple sets has been considered by Ronse (1988), Ma (1994), Kong (1995) and Gau and Kong (2003) as the basis for the definition of

$\overline{1}$ As an example, the singleton set $Y_{2}$, with the same configuration of neighbours, could appear in an object $X_{2}^{\prime}$ such that $X_{2}^{\prime} \backslash Y_{2}$ has not the same topology as $X_{2}^{\prime}$. This kind of configuration has been exposed and analysed by Morgenthaler (1981) and Fourey and Malgouyres (2003). 


\section{日司日回日}

(a)

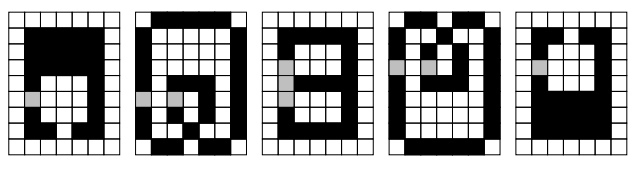

(b)

Fig. 5. (a) A discrete version $X_{1}$ of the Bing's house depicted in Fig. 2, decomposed into its five planar slices for visualisation. The set $X_{1}$ (considered in 26-adjacency) is composed of 135 points. It is topologically equivalent to any singleton $\{x\} \subset X_{1}$, but does not contain any simple point. (b) A "simple set" $Y_{1}$ (in light grey) for $X_{1}$.

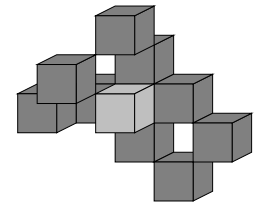

(a)

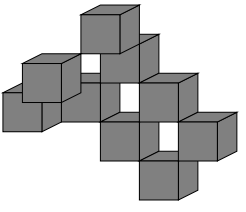

(b)

Fig. 6. (a) A set $X_{2}$ and a singleton set $Y_{2} \subset X_{2}$ (in light grey) "simple" for $X_{2}$. (b) The set $X_{2} \backslash Y_{2}$.
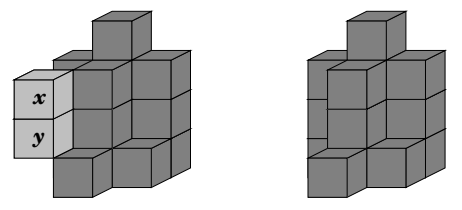

(a)
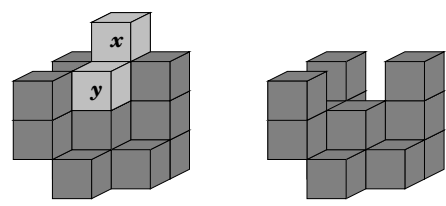

(b)
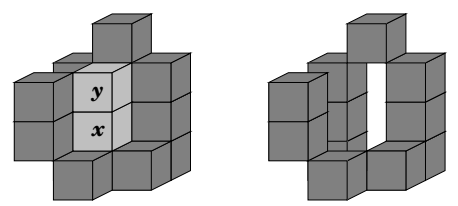

(c)

Fig. 7. A set $X_{3-5}$ including several kinds of simple sets (in light grey). (a) Left: $Y_{3} \subset X_{3-5}$, right: $X_{3-5} \backslash Y_{3}$. (b) Left: $Y_{4} \subset X_{3-5}$, right: $X_{3-5} \backslash Y_{4}$. (c) Left: $Y_{5} \subset X_{3-5}$, right: $X_{3-5} \backslash Y_{5}$. 
the notion of minimal non-simple sets (i.e. sets which are not simple while all their strict subsets are simple). In particular, these simple sets include the ones described in the previous paragraph.

The set $Y_{5}$ is composed of two points $x$ and $y$ which are both non-simple for $X_{3-5}$ (the removal of either $x$ or $y$ from $X_{3-5}$ would create a "tunnel"). However, $Y_{5}$ can be removed from $X_{3-5}$ in a monotonic way which corresponds to a reduction of $X_{3-5}$ (which will be described latter in the paper, and consisting in iteratively removing "successive parts" of $x$ and $y$ until obtaining $X_{3-5} \backslash Y_{5}$ ) without altering its topology. Such a set $Y_{5}$ is then a simple set for $X_{3-5}$, despite the fact that it does not contain any simple points. More generally, any subset $Y$ of a set $X$ (composed of simple and/or non-simple points) which may be removed from $X$ by successive topologypreserving "partial removal" of its points is a simple set. Note that sets such as $Y_{4}$ and $Y_{3}$ obviously belong to this category. The notion of simple set described in this article will be defined in this way, which generalises and extends the simple sets such as defined by Ronse (1988), Ma (1994), Kong (1995), Gau and Kong (2003) or by Bertrand (1995), since they may now be composed of simple points but also of non-simple ones.

It has to be noticed that first works on simple sets have already led to a characterisation of the ones composed of exactly two non-simple points ${ }^{2}$ in a space which models $\mathbb{Z}^{3}$, such sets being called minimal simple pairs (Passat et al. (2008)). Experimental results (see Passat et al. (2008), Appendix A) have shown that considering the notion of simple pair (and thus more generally of simple set) could lead to significant improvements of topology-preserving reduction procedures of discrete objects.

Moreover, the notion of minimal simple pair provides a counter-example to the following conjecture proposed by Kong et al. (1990).

Conjecture 3 (Kong et al. (1990), Conjecture 1, $p$. 383) Suppose $X^{\prime} \subseteq X$ are finite subsets of $\mathbb{Z}^{3}$ and $X$ is collapsible to $X^{\prime}$. Then there are sets $X_{1}, X_{2}, \ldots, X_{n}$ with $X_{1}=X, X_{n}=X^{\prime}$ and, for $0<i<n, X_{i+1}=X_{i} \backslash\left\{x_{i}\right\}$ where $x_{i}$ is a simple point of $X_{i}$.

This result emphasises the fact that simple points are not sufficient to completely deal with the problem of topology-preserving reduction of discrete images, thus justifying the study of simple sets.

The purpose of this article is to propose a definition and a preliminary study of simple sets. Although the motivations exposed in this section have been mainly illustrated in the 3-D case, and especially in $\mathbb{Z}^{3}$ (where the problems linked to the limitations of simple points have first appeared from experiments), the definitions and most of the study will be proposed in $n$-D spaces, and in the framework of

2 All the sets depicted in Fig. 3 include such a simple set. In particular, the set $Y_{5}$ is a simple set for $L_{1}$ and $L_{2}$ (which both include $X_{3-5}$ ). 
cubical complexes. This framework, promoted in particular by Kovalevsky (1989), enables to model $\mathbb{Z}^{n}\left(n \in \mathbb{N}^{*}\right)$, retrieving the main notions and results of digital topology (such as the notion of simple point), but also to define more general objects composed of cubical "parts" of various dimensions and structured on regular grids.

\section{Cubical complexes}

\subsection{Basic notions}

Intuitively, a cubical complex may be thought of as a set of elements having various dimensions (e.g. vertices, edges, squares, cubes, etc.) glued together according to certain rules. We recall in this section some basic definitions on complexes (see also Bertrand and Couprie (2008)).

Let $\mathbb{Z}$ be the set of integers. We consider the families of sets $\mathbb{F}_{0}^{1}, \mathbb{F}_{1}^{1}$, such that $\mathbb{F}_{0}^{1}=\{\{a\} \mid a \in \mathbb{Z}\}, \mathbb{F}_{1}^{1}=\{\{a, a+1\} \mid a \in \mathbb{Z}\}$. A subset $f$ of $\mathbb{Z}^{n}(n \geq 1)$ which is the Cartesian product of $m$ elements of $\mathbb{F}_{1}^{1}$ and $(n-m)$ elements of $\mathbb{F}_{0}^{1}$ is called a face or an $m$-face of $\mathbb{Z}^{n}, m$ is the dimension of $f$, and we write $\operatorname{dim}(f)=m$. We denote by $\mathbb{F}^{n}$ the set composed of all faces of $\mathbb{Z}^{n}$. Note that there is a strong link between the discrete notion of face introduced here, and the continuous one which is involved in the context of cellular complexes (see e.g. Kong (1997)), where the sets $\mathbb{F}_{0}^{1}, \mathbb{F}_{1}^{1}$ would be "replaced" by their continuous analogues $\mathbb{R}_{0}^{1}=\{[a, a] \mid a \in \mathbb{Z}\}$, $\mathbb{R}_{1}^{1}=\{] a, a+1[\quad \mid a \in \mathbb{Z}\}$. This continuous interpretation of the discrete notion of face is illustrated in Fig. 8, which justifies, in particular, the way to visually represent faces in the sequel of the article.

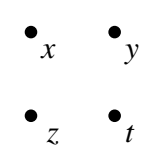

(a)

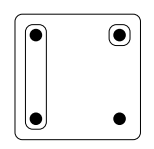

(b)

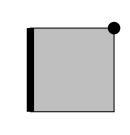

(c)

Fig. 8. Discrete and continuous interpretations of the notion of face. (a) Four elements $x=(0,1), y=(1,1), z=(0,0), t=(1,0) \in \mathbb{Z}^{2}$. (b) The 0 -face $\{y\}=\{1\} \times\{1\}$, the 1-face $\{x, z\}=\{0\} \times\{0,1\}$, and the 2 -face $\{x, y, z, t\}=\{0,1\} \times\{0,1\}$. (c) The continuous analogues of the faces of $(b)$ : the 0 -face $[1,1] \times[1,1]$, the 1 -face $[0,0] \times] 0,1[$, and the 2 -face $] 0,1[\times] 0,1[$.

Let $f$ be a face in $\mathbb{F}^{n}$. We set $\hat{f}=\left\{g \in \mathbb{F}^{n} \mid g \subseteq f\right\}$, and $\hat{f}^{*}=\hat{f} \backslash\{f\}$ (see Fig. 9). Any $g \in \hat{f}$ is a face of $f$, and any $g \in \hat{f}^{*}$ is a proper face of $f$. If $F$ is a finite set of faces of $\mathbb{F}^{n}$, we write $F^{-}=\bigcup_{f \in F} \hat{f}, F^{-}$is the closure of $F$ (see Fig. 10). We set $\operatorname{star}(f)=\left\{g \in \mathbb{F}^{n} \mid f \subseteq g\right\}$, and $\operatorname{star}^{*}(f)=\operatorname{star}(f) \backslash\{f\}, \operatorname{star}(f)$ and $\operatorname{star}^{*}(f)$ are the star and the proper star of $f$, respectively. If $F$ is a finite set of faces of $\mathbb{F}^{n}$ and $f \in F$, we set $\operatorname{star}(f, F)=\operatorname{star}(f) \cap F$, and $\operatorname{star}^{*}(f, F)=\operatorname{star}(f, F) \backslash\{f\}($ see 


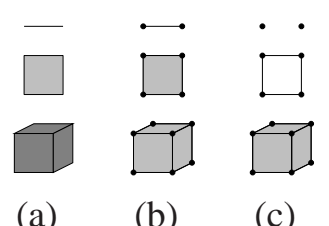

Fig. 9. (a) Three faces $f_{i}(i \in[1,3])$ of dimension $i$. (b) $\hat{f}_{i}$. (c) $\hat{f}_{i}^{*}$. Dots/lines: $0 / 1$-faces; in light grey: 2-faces; in dark grey: 3-faces.

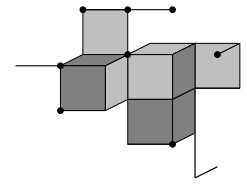

(a)

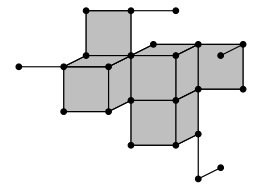

(b)

Fig. 10. (a) A set of faces $F$. (b) The closure $F^{-}$of $F$.

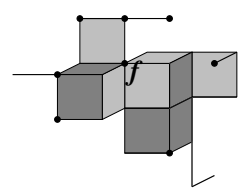

(a)

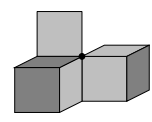

(b)

Fig. 11. (a) A set of faces $F$. (b) $\operatorname{star}(f, F)$, for the 0 -face $f \in F$.

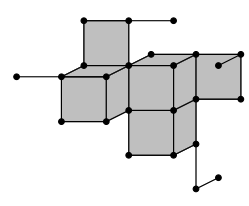

(a)

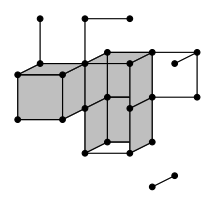

(b)

Fig. 12. (a) A complex $F$. (b) A subcomplex $G$ of $F$.

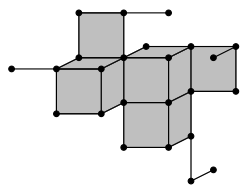

(a)

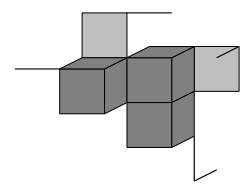

(b)

Fig. 13. (a) A complex $F$. (b) The set $F^{+} \subseteq F$ of the facets of $F$.

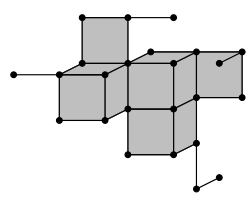

(a)

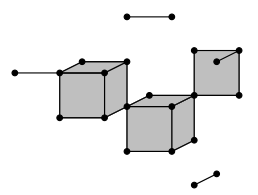

(b)

Fig. 14. (a) A complex $F$. (b) A principal subcomplex $H$ of $F$. 
Fig. 11).

A set $F$ of faces of $\mathbb{F}^{n}$ is a cell or an $m$-cell if there exists an $m$-face $f \in F$, such that $F=\hat{f}$. The boundary of a cell $\hat{f}$ is the set $\hat{f}^{*}$.

A finite set $F$ of faces of $\mathbb{F}^{n}$ is a complex (in $\mathbb{F}^{n}$ ) if for any $f \in F$, we have $\hat{f} \subseteq F$, i.e., if $F=F^{-}$. Any subset $G$ of a complex $F$ which is also a complex is a subcomplex of $F$ (see Fig. 12). If $G$ is a subcomplex of $F$, we write $G \leq F$. If $G \leq F$ and $G \neq F$, we write $G \prec F$. If $F$ is a complex in $\mathbb{F}^{n}$, we also write $F \leq \mathbb{F}^{n}$.

A face $f \in F$ is a facet of $F$ if there is no $g \in F$ such that $f \in \hat{g}^{*}$. We denote by $F^{+}$the set composed of all facets of $F$ (see Fig. 13). Observe that $\left(F^{+}\right)^{-}=F^{-}$and thus, that $\left(F^{+}\right)^{-}=F$ whenever $F$ is a complex.

If $G$ is a subcomplex of $F$, and $G^{+} \subseteq F^{+}$, then $G$ is a principal subcomplex of $F$, and we write $G \sqsubseteq F$ (see Fig. 14). If $G \sqsubseteq F$ and $G \neq F$, we write $G \sqsubset F$.

The dimension of a non-empty complex $F$ in $\mathbb{F}^{n}$ is defined by $\operatorname{dim}(F)=\max \{\operatorname{dim}(f) \mid$ $\left.f \in F^{+}\right\}$. We say that $F$ is an $m$-complex if $\operatorname{dim}(F)=m$. We say that $F$ is a pure complex if for all $f \in F^{+}$, we have $\operatorname{dim}(f)=\operatorname{dim}(F)$.

\subsection{Detachment operation}

Definition 4 Let $n \geq 1$. Let $F \leq \mathbb{F}^{n}$ be a cubical complex. Let $G \leq F$ be a subcomplex of $F$. We set $F \otimes G=\left(F^{+} \backslash G^{+}\right)^{-}$. The set $F \otimes G$ is a complex which is the detachment of $G$ from $F$ (see Fig. 15).

Definition 5 Let $n \geq 1$. Let $F \leq \mathbb{F}^{n}$ be a cubical complex. Let $G \leq F$ be a subcomplex of $F$. The attachment of $G$ to $F$ is the complex defined by $\operatorname{Att}(G, F)=$ $G \cap(F \otimes G)$ (see Fig. 16).

\section{Simple sets and minimal simple sets}

\subsection{Topology in cubical complexes}

Let $F \subseteq \mathbb{F}^{n}$ be a non-empty set of faces. A sequence $\left(f_{i}\right)_{i=0}^{s}(s \geq 0)$ of faces in $F$ is a path in $F$ (from $f_{0}$ to $f_{s}$ ) if for all $i \in[0, s-1]$, either $f_{i}$ is a face of $f_{i+1}$ or $f_{i+1}$ is a face of $f_{i}$ (with $f_{i}, f_{i+1} \in F$ ). We say that $F$ is connected if, for any two faces $f, g \in F$, there is a path in $F$ from $f$ to $g$. We say that $G$ is a connected component of $F$ if $G \subseteq F, G$ is connected and if $G$ is maximal for these two properties (i.e., we 


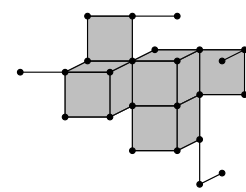

(a)

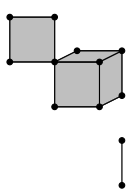

(b)

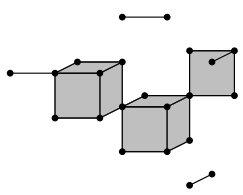

(c)

Fig. 15. (a) A complex $F$. (b) A (principal) subcomplex $G$ of $F$. (c) The detachment of $G$ from $F$.

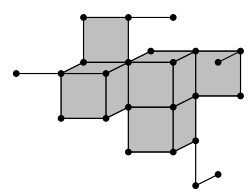

(a)

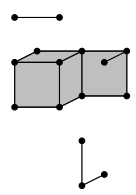

(b)

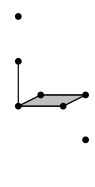

(c)

Fig. 16. (a) A complex $F$. (b) A (principal) subcomplex $G$ of $F$. (c) The attachment $\operatorname{Att}(G, F)$ of $G$ to $F$.

have $H=G$ whenever $G \subseteq H \subseteq F$ and $H$ is connected). We denote by $C[F]$ the set of all connected components of $F$. We set $C[\emptyset]=\emptyset$.

Let $F \leq \mathbb{F}^{n}$ be a cubical complex. The Euler characteristic of $F$, written $\chi(F)$, is defined by $\chi(F)=\sum_{i=0}^{n}(-1)^{i} . v_{i}$, where $v_{i}$ is the number of $i$-faces of $F$ for $i \in[0, n]$.

The Euler-Poincaré formula shows a deep link between the Euler characteristic and the Betti numbers, which are topological invariants defined from the homology groups ${ }^{3}$ of a complex.

The Betti number $b_{i}(i \in[0, n-1])$ of a complex $F \leq \mathbb{F}^{n}$ is defined as the rank of the $i$-th homology group of $F$. The Euler-Poincare formula, in the case of a complex $F \leq \mathbb{F}^{n}$, states that $\chi(F)=\sum_{i=0}^{n-1}(-1)^{i} \cdot b_{i}$.

The Euler characteristic and the Betti numbers are well-known topological invariants. In particular, the collapse operation-defined hereafter-preserves them (Kaczynski et al. (2004)).

\subsection{Collapsing}

Collapsing is a topological operation on complexes that preserves the homotopy type.

Definition 6 Let $n \geq 1$. Let $F \leq \mathbb{F}^{n}$ be a cubical complex. Let $f \in F^{+}$. If $g \in \hat{f}^{*}$ is such that $f$ is the only face of $F$ which strictly includes $g$, then we say that $g$ is a free face, that $f$ is a border face, and that the pair $(f, g)$ is a free pair for $F$

3 An introduction to homology theory can be found e.g. in Giblin (1981). 
(see Fig. 17). If $(f, g)$ is a free pair for $F$, the complex $F \backslash\{f, g\}$ is an elementary collapse of $F$.

Note that if $(f, g)$ is a free pair, then $\operatorname{dim}(g)=\operatorname{dim}(f)-1$.

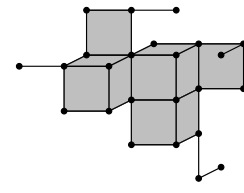

(a)

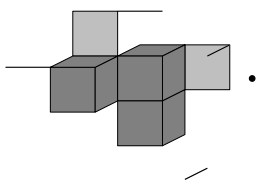

(b)

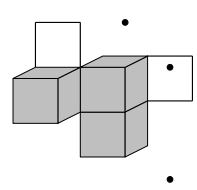

(c)

Fig. 17. (a) A complex $F$. (b) The border faces of $F$. (c) The free faces of $F$. Any pair $(f, g)$ such that $f$ is in (b) and $g \subset f$ is in (c) is a free pair for $F$.

Definition 7 Let $n \geq 1$. Let $F \leq \mathbb{F}^{n}$ be a cubical complex. Let $G \leq F$ be a subcomplex of $F$. We say that $F$ collapses onto $G$, and we note $F \searrow G$, if there exists a sequence of complexes $\left\langle F_{i}\right\rangle_{i=0}^{t}(t \geq 0)$ such that $F_{0}=F, F_{t}=G$, and $F_{i}$ is an elementary collapse of $F_{i-1}$ for all $i \in[1, t]$. The sequence $\left\langle F_{i}\right\rangle_{i=0}^{t}$ is a collapse sequence from $F$ to $G$ (see Fig. 18). Generally, we will also call collapse sequence from $F$ to $G$, the sequence $\left\langle\left(f_{i}, g_{i}\right)\right\rangle_{i=1}^{t}$ of free pairs verifying $F_{i}=F_{i-1} \backslash\left\{f_{i}, g_{i}\right\}$ for all $i \in[1, t]$.

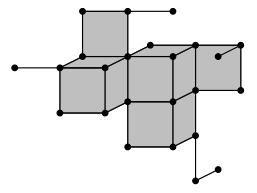

(a)

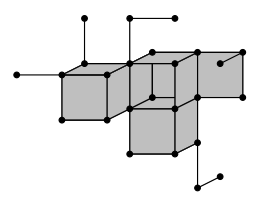

(c)

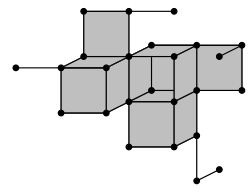

(b)

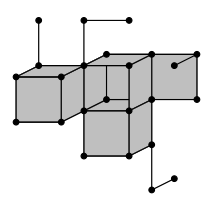

(d)

Fig. 18. From (a) to (d): a collapse sequence from $F$ to a subcomplex $K \leq F$.

Remark 8 The collapse operation, defined by Whitehead (1938), is a classical notion in combinatorial topology. It can be seen as a discrete counterpart of a deformation retraction defined on "continuous" complexes (see Fig. 19). It was introduced in the field of image (analysis and) processing by Kong (1997) in order to establish a link between the notions of simple point and continuous deformation.

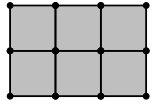

(a)

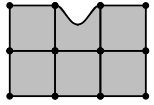

(b)

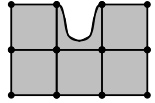

(c)

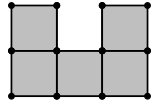

(d)

Fig. 19. (a) A (2-)complex $F$. (d) An elementary collapse $G$ of $F$. (a-d) Some steps of the "continuous" deformation retraction implicitly associated to this elementary collapse. 


\subsection{Simple cells and lumps}

Intuitively a cell $G \leq F$ is called simple if there is a topology-preserving deformation of $F$ over itself onto the relative complement of $G$ in $F$. The following definition of simple cells, based on the collapse operation, can be seen as a discrete counterpart of the one given by Kong (1997).

Definition 9 Let $n \geq 1$. Let $F \leq \mathbb{F}^{n}$ be a cubical complex. Let $f \in F$ be a face of $F$. The cell $\hat{f}$ is a simple cell for $F$ if $F \searrow F \otimes \hat{f}$.

From the notion of simple cell, it is now possible to define the notion of simpleequivalence, and then the notion of lump in the framework of cubical complexes.

Definition 10 Let $n \geq 1$. Let $F, F^{\prime} \leq \mathbb{F}^{n}$ be two cubical complexes. We say that $F$ and $F^{\prime}$ are simple-equivalent if there exists a sequence of complexes $\left\langle F_{i}\right\rangle_{i=0}^{t}(t \geq 0)$ such that $F_{0}=F, F_{t}=F^{\prime}$, and for any $i \in[1, t]$, we have either:

(i) $F_{i}=F_{i-1} \otimes H_{i}$, where $H_{i} \leq F_{i-1}$ is a simple cell for $F_{i-1}$; or

(ii) $F_{i-1}=F_{i} \otimes H_{i}$, where $H_{i} \leq F_{i}$ is a simple cell for $F_{i}$.

The simple-equivalence enables to establish a notion of topology preservation which is not only based on monotonic deformations, i.e. on reductions or growings based on simple cells (generally considered for applicative purpose (Dokládal et al. (1999); Passat et al. (2005))), but also on non-monotonic ones. Two objects which are simple-equivalent (see e.g. the objects of Figs. 6(a) and (b)) can be obtained by both removal and addition of simple cells during the deformation process. Consequently, their topologies are similar since the homotopy type of two intermediate objects generated during the deformation process is the same. In particular, it may happen that an object $F$ and one of its strict subsets $G$ are simple-equivalent while there is no way to obtain $G$ from $F$ by successively removing simple cells (see e.g. the complexes depicted in Fig. 3). Such objects $F$ are called lumps.

Definition 11 Let $n \geq 1$. Let $F \leq \mathbb{F}^{n}$ be a cubical complex. Let $F^{\prime} \leq F$ be a subcomplex of $F$ such that $F$ and $F^{\prime}$ are simple-equivalent. If $F$ does not include any principal simple cell outside $F^{\prime}$, then we say that $F$ is a lump relative to $F^{\prime}$, or simply a lump.

As stated in Section 2, a lump $F$ relative to $F^{\prime}$, although not including any simple cell which can be detached to provide a monotonic reduction strictly converging onto $F^{\prime}$, may however include some subsets which can be detached in such a way. Such simple sets, defined in the next section, then enable to develop monotonic (and then algorithmically tractable) reduction processes which can be more efficient than those only based on simple cells (since they can "break" some lumps). 


\subsection{Simple sets}

Definition 9 proposed for simple cells naturally extends to subcomplexes $G \leq F$ which contain an arbitrary number of facets, leading to the notion of simple set.

Definition 12 Let $n \geq 1$. Let $F \leq \mathbb{F}^{n}$ be a cubical complex. Let $G \leq F$ be a subcomplex of $F$. We say that $G$ is simple for $F$ if $F \searrow F \otimes G$. Such a subcomplex $G$ is called a simple subcomplex of $F$ or a simple set for $F$.

Note that the notion of attachment leads to the following local characterisation of simple sets.

Proposition 13 (Bertrand (2007)) Let $n \geq 1$. Let $F \leq \mathbb{F}^{n}$ be a cubical complex. Let $G \leq F$ be a subcomplex of $F$. The complex $G$ is simple for $F$ if and only if $G \searrow \operatorname{Att}(G, F)$.

The following notion of removable set enables to model the part of a complex which is effectively deleted during a collapse sequence.

Definition 14 Let $n \geq 1$. Let $F \leq \mathbb{F}^{n}$ be a cubical complex. Let $G \subseteq F$ be a subset of $F$. We say that $G$ is removable for $F$, or that $G$ is a removable set for $F$, if $F \backslash G \leq F$ and $F \searrow F \backslash G$.

This notion is strongly related to the one of simple set, since a simple set can be partitioned into two subsets: its attachment and its removable part. More formally, we have the following property, which directly derives from the definitions.

Property 15 Let $n \geq 1$. Let $F \leq \mathbb{F}^{n}$ be a cubical complex. Let $G \leq F$ be a subcomplex of $F$. Then, $G$ is simple for $F$ if and only if $G \backslash \operatorname{Att}(G, F)$ is removable for $F$.

Definition 16 Let $n \geq 1$. Let $F \leq \mathbb{F}^{n}$ be a cubical complex. Let $G \leq F$ be a subcomplex of $F$. We say that $G$ is trivially simple for $F$, or that $G$ is a trivial simple set for $F$, if $G$ is simple for $F$ while its removable part is empty (i.e. $G \backslash \operatorname{Att}(G, F)=$ $\emptyset)$.

Remark 17 If $G$ is a trivial simple set for $F$, then $F \otimes G=F$. As such sets do not present a real interest from an algorithmic point of view, from now on, we will always implicitly consider that a simple set $G$ for $F$ is non-trivial. Note that in this context, a simple cell for $F$ is necessarily a principal cell of $F$.

We introduce now the notion of minimal simple set.

Definition 18 Let $n \geq 1$. Let $F \leq \mathbb{F}^{n}$ be a cubical complex. Let $G \leq F$ be a subcomplex of $F$. The complex $G$ is a minimal simple subcomplex of (or a minimal simple set for) $F$ if $G$ is a (non-trivial) simple set for $F$ and $G$ is minimal (with 
respect to $\leq$ ) for this property (i.e. $\forall H \leq G, H$ is (non-trivially) simple for $F$ $\Rightarrow H=G)$.

The notion of minimal simple set may be useful from both theoretical and algorithmic points of view since $(i)$ the existence of a simple set necessarily implies the existence of at least one minimal simple set, and (ii) by definition, a minimal simple set is necessarily easier (or, at least, not harder) to characterise than a "general" simple set. In particular, we can hope that in several cases (depending on the dimension(s) of $\mathbb{F}^{n}$ and/or of $F$, for example), the study of minimal simple sets could be sufficient to deal with the problem of detaching all simple sets from a complex (an example of this assertion will be found e.g. in Mazo and Passat (2009)).

\section{General properties of simple sets}

In this section, we present and prove some properties of simple and minimal simple sets valid in any dimension $n \geq 1$. We first consider "global" properties related to their principalness with respect to their superset (Section 5.1), to their removable part (Section 5.2), to their attachment (Section 5.3) and to their connectedness (Section 5.4). More "local" properties dealing with 0-D configurations of their attachment (Section 5.5) are then considered.

The purpose of Sections 5 and 6 is in particular to obtain a description as accurate as possible of (minimal) simple sets, enabling to further derive characterisations of subfamilies of such sets, and more generally to improve the algorithmic cost of their detection and removal.

\subsection{Principalness}

Proposition 19 (which is illustrated in Fig. 20) implies that, from an algorithmic point of view, the study of the simple sets of a complex can be restricted to the family of its principal subcomplexes, thus leading to a strong decrease of the number of potential simple sets to consider. In particular, minimal simple sets are principal subcomplexes, as stated in Proposition 20.

Proposition 19 Let $n \geq 1$. Let $F \leq \mathbb{F}^{n}$ be a cubical complex. Let $G \leq F$ be a simple set for $F$. Let $H \leq G$ be the maximal (with respect to $\leq$ ) subcomplex of $G$ verifying $H \sqsubseteq F$ (i.e. $\forall K \preceq G, K \sqsubseteq F \Rightarrow K \sqsubseteq H$ ). Then $H$ is a simple set for $F$ and $F \otimes H=F \otimes G$.

Proof Let $H=\left(G^{+} \cap F^{+}\right)^{-} \leq G$. By construction, we have $H \neq \emptyset$ (as $G$ is not trivial, see Remark 17), $H \sqsubseteq F$ and $H$ is the maximal (with respect to $\leq$ ) subcomplex of $G$ for this property. Moreover, we have $F \otimes H=\left(F^{+} \backslash H^{+}\right)^{-}=\left(F^{+} \backslash\left(G^{+} \cap F^{+}\right)\right)^{-}=$ 
$\left(F^{+} \backslash G^{+}\right)^{-}=F \otimes G$. Finally, it comes $F \searrow F \otimes G=F \otimes H$, and $H$ is then a simple set for $F$.

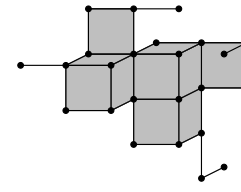

(a)

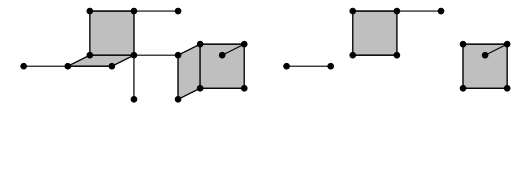

(b)

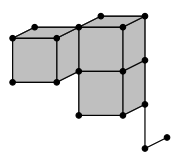

(d)

Fig. 20. (a) A 3-complex $F$. (b) A simple set $G \leq F$ of $F$. (c) The maximal (with respect to $\leq$ ) subcomplex $H$ of $G$ verifying $H \sqsubseteq F ; H$ is a simple set for $F$. (d) The detachment of $H$ from $F$, which is equal to the detachment of $G$ from $F$ (see Proposition 19).

Proposition 20 Let $n \geq 1$. Let $F \leq \mathbb{F}^{n}$ be a cubical complex. Let $G \leq F$ be a minimal simple set for $F$. Then $G \sqsubseteq F$.

Proof Since $G$ is a simple set for $F$, from Proposition 19, there exists $H \leq G$ such that $H \sqsubseteq F$ and $H$ is a simple set for $F$. The minimality of $G$ implies that $H=G$. Then, $G \sqsubseteq F$.

Remark 21 From now on, the simple sets of a complex $F$ will be considered among the complexes $G \sqsubseteq F$. Moreover, if $G$ is a simple set for $F$, then we obviously have $F \odot G \neq \emptyset$, i.e. $G^{+} \subset F^{+}$, and thus, $G \prec F$. Based on these considerations, given a complex $F \leq \mathbb{F}^{n}$, we will only consider simple sets $G$ of $F$ which verify $(\emptyset \sqsubset) G \sqsubset F$.

\subsection{Removable part}

The following two propositions, related to the connectedness properties of the removable part of simple sets, will be essentially used to establish the results of Sections 5.3 and 5.4 .

Proposition 22 Let $n \geq 1$. Let $F \leq \mathbb{F}^{n}$ be a cubical complex. Let $G \sqsubset F$ be a simple set for $F$. Let $G_{r}=G \backslash \operatorname{Att}(G, F)$ be the removable part of $G$. Let $H_{r} \subseteq G_{r}$ be a connected component of $G_{r}$. If $H_{r} \neq G_{r}$ (i.e. if $H_{r} \subset G_{r}$ ), then:

(i) $H=H_{r}^{-} \sqsubset G \sqsubset F$ is a simple set for $F$;

(ii) $G \otimes H$ is a simple set for $F \otimes H$.

Proof Let us suppose that $H_{r} \neq G_{r}$. Let $\left\langle\left(f_{i}, g_{i}\right)\right\rangle_{i=1}^{t}(t \geq 2)$ be a collapse sequence from $F$ to $F \otimes G$. Let $j \in[2, t]$ such that $f_{j} \in H_{r}$ and $f_{j-1} \notin H_{r}$, if such faces exist. As $H_{r}$ is a connected component of $G_{r}$, we also have $g_{j} \in H_{r}$ while $g_{j-1} \notin H_{r}$. The pairs $\left(f_{j-1}, g_{j-1}\right)$ and $\left(f_{j}, g_{j}\right)$ can then obviously be exchanged, providing another valid collapse sequence from $F$ to $F \otimes G$. We can then inductively build a new collapse sequence $\left\langle\left(f_{i}, g_{i}\right)\right\rangle_{i=1}^{t}$ verifying $f_{i} \notin H_{r} \Rightarrow f_{i+1} \notin H_{r}$ for all $i \in[1, t-1]$. Let $\alpha=\max \left\{i \in[1, t] \mid f_{i} \in H_{r}\right\}$. By definition of $\left\langle\left(f_{i}, g_{i}\right)\right\rangle_{i=1}^{t}$ and $\alpha$, we have $f_{i} \in H_{r}$ for all $i \in[1, \alpha]$ while $f_{i} \notin H_{r}$ for all $i \in[\alpha+1, t]$. Moreover, for the same reason 
as previously, we have $g_{i} \in H_{r}$ for all $i \in[1, \alpha]$ while $g_{i} \notin H_{r}$ for all $i \in[\alpha+1, t]$. Since $H_{r} \subseteq\left\{f_{i}, g_{i}\right\}_{i=1}^{t}$, we then have $H_{r}=\left\{f_{i}, g_{i}\right\}_{i=1}^{\alpha}$. The sequence $\left\langle\left(f_{i}, g_{i}\right)\right\rangle_{i=1}^{\alpha}$ is then a collapse sequence from $F$ to $F \backslash H_{r}$. Let $H=H_{r}^{-}$. Since $H_{r}\left(\neq G_{r}\right)$ is a connected component of $G_{r}=G \backslash \operatorname{Att}(G, F)$ where $G \sqsubset F$ is a simple set for $F$, we have in particular $H \sqsubset G \sqsubset F$. Moreover, $H_{r}=H \backslash \operatorname{Att}(H, F)$, and then, $F \backslash H_{r}=F \otimes H$. Consequently, we have $F \searrow F \otimes H$, i.e. $H \sqsubset F$ is a simple set for $F$. By definition, $\left\langle\left(f_{i}, g_{i}\right)\right\rangle_{i=\alpha+1}^{t}$ is a collapse sequence from $F \otimes H$ to $F \otimes G$. As $H \sqsubset G \sqsubset F$, we have $G \otimes H \sqsubset F \otimes H$, and $F \otimes G=(F \otimes H) \otimes(G \otimes H)$. Then, we finally obtain $F \otimes H \searrow(F \odot H) \otimes(G \otimes H)$, i.e. $G \otimes H$ is a simple set for $F \otimes H$.

From Proposition 22, we immediately derive the following proposition.

Proposition 23 Let $n \geq 1$. Let $F \leq \mathbb{F}^{n}$ be a cubical complex. Let $G \sqsubset F$ be a minimal simple set for $F$. Let $G_{r}=G \backslash \operatorname{Att}(G, F)$ be the removable part of $G$. Then, $G_{r}$ is connected.

\subsection{Attachment}

Proposition 24 (which is illustrated in Fig. 21(a-e)) enables to restrict the research of simple sets and authorises in particular the development of region-growing (and possibly parallel) strategies.

Proposition 24 Let $n \geq 1$. Let $F \leq \mathbb{F}^{n}$ be a cubical complex. Let $G \sqsubset F$ be a simple set for $F$. Let $H \sqsubset G$ be a strict principal subcomplex of $G$. If $\operatorname{Att}(H, F) \subseteq \operatorname{Att}(G, F)$, then:

(i) $H$ is a simple set for $F$;

(ii) $G \otimes H$ is a simple set for $F \otimes H$.

Proof Let $G_{r}=G \backslash \operatorname{Att}(G, F)$ be the removable part of $G$. Let $H_{r}=H \backslash \operatorname{Att}(H, F)$. Let $K_{r}$ be a connected component of $H_{r}$, in particular, we have $K_{r} \subseteq G_{r}$. As $K_{r}$ is connected, it is included in a connected component of $G_{r}$. Let $K=K_{r}^{-}$. We have $\operatorname{Att}(K, F) \subseteq \operatorname{Att}(H, F) \subseteq \operatorname{Att}(G, F)$, then $K_{r}$ is necessarily a connected component of $G_{r}$. Since $\operatorname{Att}(H, F) \subseteq \operatorname{Att}(G, F)$, we have $H_{r} \subseteq G_{r}$, and as $H \sqsubset G$, we obtain $H_{r} \neq G_{r}$. Then, from Proposition 22, $K \sqsubset G \sqsubset F$ is a simple set for $F$, and $G \otimes K$ is a simple set for $F \otimes K$. If $H=K$, then the result holds. Otherwise, since $H \otimes K \sqsubset G \otimes K$, the result easily follows by induction on the connected components of $H_{r}$. 


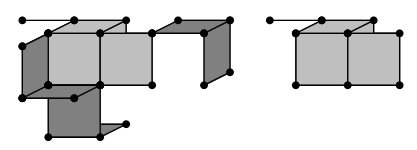

(a)

(b)

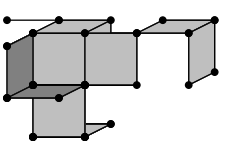

(c)

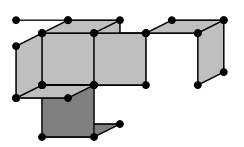

(d)

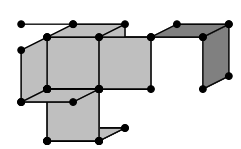

(e)

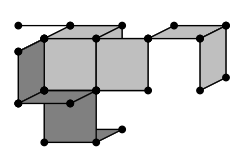

(f)

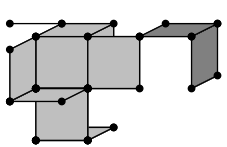

(g)

Fig. 21. (a) A complex $F$ and a simple set $G \sqsubset F$ (in dark grey). (b) The detachment of $G$ from $F$. (c-e) Three strict principal subcomplexes $H$ of $G$ such that $\operatorname{Att}(H, F) \subseteq \operatorname{Att}(G, F)$ (in dark grey). (f,g) The two connected components $H$ of $G$ (in dark grey). The detachment of $G$ from $F$ (b) can be obtained from $F$ (a) by detaching the subcomplexes $H$ of (c-e) (resp. the subcomplexes $H$ of $(\mathrm{f}, \mathrm{g})$ ) in any order or in parallel (see Proposition 24 (resp. Proposition 25)).

\subsection{Connectedness}

Proposition 25 (which is illustrated in Fig. 21(a,f-g)) is a specific case of Proposition 24. It establishes the connectedness of the simple sets which have to be searched to enable the removal of all of them. This proposition, weaker than Propositions 22 and 24, is however more easily usable for the definition of region-growing strategies.

Proposition 25 Let $n \geq 1$. Let $F \leq \mathbb{F}^{n}$ be a cubical complex. Let $G \sqsubset F$ be a non-connected simple set for $F$. Let $H \sqsubset G$ be a connected component of $G$. Then:

(i) $H$ is a simple set for $F$;

(ii) $G \otimes H$ is a simple set for $F \otimes H$.

The next proposition derives from Proposition 25. It is also a direct consequence of Proposition 23.

Proposition 26 Let $n \geq 1$. Let $F \leq \mathbb{F}^{n}$ be a cubical complex. Let $G \sqsubset F$ be a minimal simple set for $F$. Then $G$ is connected.

Since the collapse operation does not alter the connectedness of complexes, we derive from Propositions 13 and 26 the following result.

Proposition 27 Let $n \geq 1$. Let $F \leq \mathbb{F}^{n}$ be a cubical complex. Let $G \sqsubset F$ be a minimal simple set for $F$. Then $\operatorname{Att}(G, F)$ is connected. 
Remark 28 More generally, as the collapse operation does not alter the Betti numbers of a complex, the Betti numbers of $G \sqsubset F$ and $A t t(G, F)$ are equal if $G$ is a simple (and a fortiori a minimal simple) set for $F$.

\subsection{The case of 0-D attachments}

The following lemma will be necessary to prove Proposition 30 .

Lemma 29 Let $n \geq 1$. Let $F \leq \mathbb{F}^{n}$ be a cubical complex. Let $h \in F$ be a 0 -face and $C$ be a connected component of $\operatorname{star}^{*}(h, F)$. Let $\left\langle\left(f_{i}, g_{i}\right)\right\rangle_{i=1}^{t}(t \geq 1)$ be a collapse sequence for $F$. If there exists a path $\pi=\left(p_{i}\right)_{i=0}^{k}(k \geq 5)$ in $F$ such that $p_{0}=p_{k}=h$, $p_{1} \in C, p_{k-1} \notin C$ and $p_{i} \notin \operatorname{star}(h, F)$ for all $i \in[2, k-2]$, then there exists a path $\pi^{\prime}=\left(q_{i}\right)_{i=0}^{l}(l \geq 5)$ in $F \backslash \bigcup_{i=1}^{t}\left\{f_{i}, g_{i}\right\}$ such that $q_{0}=q_{l}=h, q_{1} \in C, q_{l-1} \notin C$, and $q_{i} \notin \operatorname{star}(h, F)$ for all $i \in[2, l-2]$.

Proof Without loss of generality, we can assume that $\pi$ is such that $\max \left\{\operatorname{dim}\left(p_{i}\right) \mid\right.$ $i \in[0, k]\}=1$, and all the 1-faces of $\pi$ are distinct (remember that $p_{1} \in C$ and $\left.p_{k-1} \notin C\right)$. If $\left\{p_{i}\right\}_{i=0}^{k} \cap\left\{f_{1}, g_{1}\right\}=\emptyset$, we set $\pi^{\prime}=\pi$. Otherwise, since by our previous assumption, $\pi$ has no free 0 -face in $F$, there exists a 1 -face $p_{\alpha}(\alpha \in[1, k-1])$ of $\pi$ such that $g_{1}=p_{\alpha}$. We can clearly replace $p_{\alpha}$ in $\pi$ by a sequence $\sigma$ of the 5 faces of $\hat{f}_{1}^{*} \backslash\left\{p_{\alpha-1}, p_{\alpha}, p_{\alpha+1}\right\}$ (in the correct order) to obtain a new path $\pi^{\prime}=\left(q_{i}\right)_{i=1}^{t+4}=\left(q_{i}\right)_{i=1}^{l}$ in $F \backslash\left\{f_{1}, g_{1}\right\}$ from $h$ to $h$. Note that the first (resp. last) 1-face $q_{\alpha}$ (resp. $\left.q_{\alpha+4}\right)$ of $\sigma$ belongs to the same connected component of $\operatorname{star}^{*}\left(p_{\alpha-1}, F\right)\left(\right.$ resp. $\left.\operatorname{star}^{*}\left(p_{\alpha+1}, F\right)\right)$ $\left(p_{\alpha-1}, p_{\alpha+1}\right.$ are 0 -faces) as $f_{1}$ and two 1-faces of $\sigma$ surrounding a 0 -face $g$ of $\sigma$ belong to the same connected component of $\operatorname{star}^{*}(g, F)$. Hence, it is easy to check that $q_{1} \in C$ and $q_{l-1} \notin C$, and if a 0 -face $q_{j}(j \in[4, t])$ of $\pi$ is equal to $h$, one of the two paths $\left(q_{i}\right)_{i=1}^{j},\left(q_{i}\right)_{i=j}^{t+4}$ verifies all the required properties (otherwise, $\left(q_{i}\right)_{i=1}^{t+4}$ verifies the same required properties). As the connected component of $\operatorname{star}^{*}\left(h, F \backslash\left\{f_{1}, g_{1}\right\}\right)$ containing $q_{1}$ is included in $C$, the result follows by induction.

Proposition 30 Let $n \geq 1$. Let $F \leq \mathbb{F}^{n}$ be a cubical complex. Let $G \sqsubset F$ be a simple set for $F$. Let $f \in G$ be a 0 -face of $G$ such that $\operatorname{star}^{*}(f, F)$ is not connected, and $C\left[\operatorname{star}^{*}(f, F)\right] \cap C\left[\operatorname{star}^{*}(f, G)\right] \neq \emptyset$ (see Fig. 22(d,g,h)). Then:

(i) $\exists H_{r} \in C[G \backslash\{f\}]$ such that $H_{r} \cap \operatorname{Att}(G, F)=\emptyset$;

(ii) $\forall H_{r} \in C[G \backslash\{f\}]$ such that $H_{r} \cap \operatorname{Att}(G, F)=\emptyset$, we have:

(a) $H_{r} \cap \operatorname{star}^{*}(f, F) \in C\left[\operatorname{star}^{*}(f, F)\right]$;

(b) $H_{r} \cap \operatorname{star}^{*}(f, F) \in C\left[\operatorname{star}^{*}(f, G)\right]$.

Proof The proof is proposed for $F$ and $G$ connected. It straightforwardly extends to $F$ and/or $G$ non-connected (see Proposition 25) by considering the connected components of $F$ and $G$ containing $f$.

Proof of $(i)$. Let $A \in C\left[\operatorname{star}^{*}(f, F)\right] \cap C\left[\operatorname{star}^{*}(f, G)\right]$. Let $H_{r} \in C[F \backslash\{f\}]$ such that $A \subset H_{r}$. Since $F \searrow F \otimes G$, we deduce that $H_{r} \cap \operatorname{star}^{*}(f, F)=H_{r} \cap \operatorname{star}^{*}(f, G)=A$ 
(otherwise, by Lemma 29, we would have a path in $F \otimes G$ including a face of A). If $H_{r} \cap \operatorname{Att}(G, F)=\emptyset$, then we have $H_{r} \in C[G \backslash\{f\}]$, and we are done. Let us now suppose that $H_{r} \cap \operatorname{Att}(G, F) \neq \emptyset$. As the collapse operation preserves connectedness, and since $G$ is connected, $\operatorname{Att}(G, F)$ is also connected. Then, we have $\operatorname{Att}(G, F) \subset$ $H_{r}$ (otherwise, $f \in \operatorname{Att}(G, F)$, and then $A \cap \operatorname{Att}(G, F) \neq \emptyset$, in contradiction with the fact that $\left.A \in C\left[\operatorname{star}^{*}(f, F)\right] \cap C\left[\operatorname{star}^{*}(f, G)\right]\right)$. Consequently, it comes $f \notin \operatorname{Att}(G, F)$, and as $\operatorname{star}^{*}(f, F)$ is not connected, there exists $B \in C\left[\operatorname{star}^{*}(f, F)\right] \cap C\left[\operatorname{star}^{*}(f, G)\right]$, with $B \neq A$. Let $H_{r}^{\prime} \in C[F \backslash\{f\}]$ such that $B \subset H_{r}^{\prime}$. As $H_{r}^{\prime} \cap H_{r}=\emptyset$, we have $H_{r}^{\prime} \cap \operatorname{Att}(G, F)=\emptyset$ and $H_{r}^{\prime} \in C[G \backslash\{f\}]$. Hence $(i)$ holds.

Proof of (ii). Let $H_{r} \in C[G \backslash\{f\}]$ such that $H_{r} \cap \operatorname{Att}(G, F)=\emptyset$. Let $H=H_{r}^{-}=$ $H_{r} \cup\{f\}$. If $\operatorname{star}^{*}(f, H)$ is not connected, then from Lemma 29, we deduce that $H_{r} \cap(F \odot G) \neq \emptyset$, in contradiction with the fact that $H_{r} \cap \operatorname{Att}(G, F)=\emptyset$.

Proposition 31 Let $n \geq 1$. Let $F \leq \mathbb{F}^{n}$ be a cubical complex. Let $G \sqsubset F$ be a simple set for $F$. Let $f \in G$ be a 0 -face of $G$ such that $\operatorname{star}^{*}(f, F)$ is not connected, and $C\left[\operatorname{star}^{*}(f, F)\right] \cap C\left[\operatorname{star}^{*}(f, G)\right] \neq \emptyset$. Let $H_{r} \in C[G \backslash\{f\}]$ such that $H_{r} \cap \operatorname{Att}(G, F)=\emptyset$. Then:

(i) $H=H_{r}^{-} \sqsubseteq G \sqsubset F$ is a simple set for $F$;

(ii) $\operatorname{Att}(H, F)=\hat{f}$;

(iii) if $H \neq G$, then $G \otimes H$ is a simple set for $F \otimes H$.

Proof The proof is proposed for $F$ and $G$ connected. It straightforwardly extends to $F$ and/or $G$ non-connected (see Proposition 25) by considering the connected components of $F$ and $G$ containing $f$. Note that from Proposition 30, $H_{r}$ exists. Let $H=H_{r}^{-}=H_{r} \cup\{f\}$, we have in particular $H \sqsubseteq G \sqsubset F$ and $\operatorname{Att}(H, F)=\hat{f}$. If $f \in \operatorname{Att}(G, F)$, the result follows from Proposition 22. We now suppose that $f \notin \operatorname{Att}(G, F)$. Let $\left\langle\left(f_{i}, g_{i}\right)\right\rangle_{i=1}^{t}(t \geq 1)$ be a collapse sequence from $F$ to $F \otimes G$. Let $\alpha \in[1, t]$ be such that $g_{\alpha}=f$. Note that for all $i \in[1, \alpha-1]$, since $\operatorname{Att}(H, F)=\{f\}$, we have either $f_{i}, g_{i} \in H_{r}$ or $f_{i}, g_{i} \notin H_{r}$. Let $j \in[2, \alpha-1]$ be such that $f_{j}, g_{j} \in H_{r}$ while $f_{j-1}, g_{j-1} \notin H_{r}$, if such faces exist. The pairs $\left(f_{j-1}, g_{j-1}\right),\left(f_{j}, g_{j}\right)$ can then be exchanged, providing another valid collapse sequence from $F$ to $F \otimes G$. Moreover, as $G \backslash H_{r}$ is connected, and as the collapse operation preserves connectedness, $\left(G \backslash H_{r}\right) \backslash\left\{f_{i}, g_{i}\right\}_{i=1}^{\alpha-1}$ is connected, and then, we cannot have $f_{\alpha} \in H_{r}$ (otherwise, $(G \backslash$ $\left.H_{r}\right) \backslash\left\{f_{i}, g_{i}\right\}_{i=1}^{\alpha-1}=\{f\}$ : contradiction). As $H$ is connected, we also have $H \backslash\left\{f_{i}, g_{i}\right\}_{i=1}^{\alpha-1}$ connected, and then, $H \backslash\left\{f_{i}, g_{i}\right\}_{i=1}^{\alpha-1}=\{f\}$, then for all $i \in[\alpha, t]$, we have $f_{i}, g_{i} \notin H_{r}$. It is then possible to inductively build a new collapse sequence $\left\langle\left(f_{i}, g_{i}\right)\right\rangle_{i=1}^{t}$ verifying $f_{i} \notin H_{r} \Rightarrow f_{i+1} \notin H_{r}$ for all $i \in[1, t-1]$. Let $\beta=\max \left\{i \in[1, t] \mid f_{i} \in H_{r}\right\}$. Then, we have $\left\{f_{i}, g_{i}\right\}_{i=1}^{\beta}=H_{r}$, in particular, $H \backslash\left\{f_{i}, g_{i}\right\}_{i=1}^{\beta}=\{f\}$, and $\left\langle\left(f_{i}, g_{i}\right)\right\}_{i=1}^{\beta}$ is then a collapse sequence from $H \sqsubseteq G \sqsubset F$ to $\{f\}=\operatorname{Att}(H, F)$. Consequently, $H$ is a simple set for $F$. Moreover, if $H \neq G$, we have $\emptyset \neq G \otimes H \sqsubset F \otimes H$, and $\left\langle\left(f_{i}, g_{i}\right)\right\rangle_{i=\beta+1}^{t}$ is a collapse sequence from $F \otimes H$ to $F \otimes G=(F \otimes H) \otimes(G \otimes H)$. Hence the result holds.

Remark 32 Propositions 30 and 31 (which are illustrated in Fig. 22) enable to re- 
strict the research of simple sets to those which do not present any "isthmuses" (i.e. 0-faces satisfying the hypothesis of $f$ in Propositions 30 and 31). For instance, the face $f$ is (resp. is not) such an isthmus in Fig. 22(g,h) (resp. Fig. 22(d)). Propositions 31 establishes, in particular, that any simple set $G$ of a complex $F$ can be fully detached from $F$ by iterative (and non-deterministic) detachment of its principal subcomplexes attached to $F$ by such an isthmus.

The following proposition immediately derives from Propositions 30 and 31.

Proposition 33 Let $n \geq 1$. Let $F \leq \mathbb{F}^{n}$ be a cubical complex. Let $G \sqsubset F$ be a minimal simple set for $F$. Then, there exists at most one 0 -face $f \in G$ such that $\operatorname{star}^{*}(f, F)$ is not connected and $C\left[\operatorname{star}^{*}(f, F)\right] \cap C\left[\operatorname{star}^{*}(f, G)\right] \neq \emptyset$. If $f$ exists, then we have $\operatorname{Att}(G, F)=\{f\}$, and $\operatorname{star}^{*}(f, G)$ is connected.

\section{Properties of simple sets related to dimensions 0 and 1}

From the previous section, we can derive some properties related to $0-\mathrm{D}$ and 1-D structures of simple and minimal simple sets in $\mathbb{F}^{n}$.

\section{$6.10-D$ properties}

The first proposition is elementary.

Proposition 34 Let $n \geq 1$. Let $F \leq \mathbb{F}^{n}$ be a cubical complex. Let $G \sqsubset F$ be a simple cell for $F$. Then $\operatorname{dim}(G)>0$.

Based on this result and on the propositions of Section 5, we can prove the nonexistence of 0-facets in simple sets, and then the non-existence of 0-D simple sets.

Proposition 35 Let $n \geq 1$. Let $F \leq \mathbb{F}^{n}$ be a cubical complex. Let $G \sqsubset F$ be a simple set for $F$. Let $f \in G^{+}$be a facet of $G$. Then $\operatorname{dim}(f)>0$.

Proof If $\operatorname{dim}(f)=0$, then the cell $\hat{f}$ is a connected component of $G$. Proposition 25 then implies that $\hat{f}$ is simple for $F$, in contradiction with Proposition 34 .

The following result is an easy consequence of Proposition 35

Proposition 36 Let $n \geq 1$. Let $F \leq \mathbb{F}^{n}$ be a cubical complex. Let $G \sqsubset F$ be a simple set for $F$. Then, $\operatorname{dim}(G)>0$. 


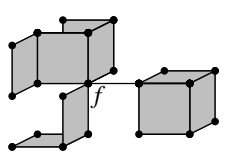

(a)

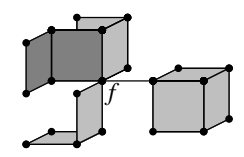

(d)

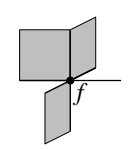

(b)

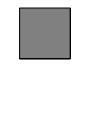

(e)

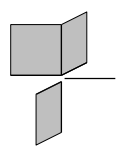

(c)

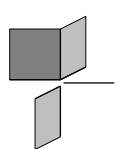

(f)

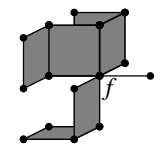

(g)

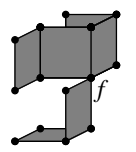

(h)

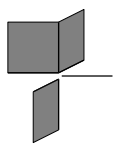

(i)

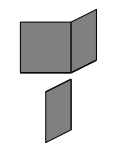

(j)

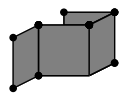

(k)

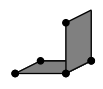

(n)

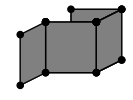

(l)

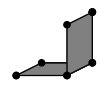

(o)

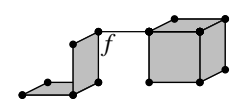

(m)

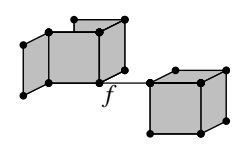

(p)

Fig. 22. (a) A complex $F$ and a 0 -face $f \in F$ such that $\operatorname{star}^{*}(F, f)$ is not connected. (b) $\operatorname{star}^{*}(F, f) \cup\{f\}$. (c) $\operatorname{star}^{*}(F, f)$, composed of three connected components. (d) In dark grey: a simple set $G \sqsubset F$ of $F$ such that $C[\operatorname{star}(f, F)] \cap C\left[\operatorname{star}^{*}(f, G)\right]=\emptyset$ : the only connected component of $\operatorname{star}^{*}(f, G)$ (e) is strictly included in one of the three connected components of $\operatorname{star}^{*}(f, F)$ (f). This set does not satisfy the hypotheses of Propositions 30 and 31. (g,h) Two simple sets $G \sqsubset F$ of $F$ such that $C\left[\operatorname{star}^{*}(f, F)\right] \cap C\left[\operatorname{star}^{*}(f, G)\right] \neq \emptyset$ and $(\mathrm{i}, \mathrm{j})$ their associated set of faces $\operatorname{star}^{*}(G, f)$ composed of three (i) and two (j) connected components. (k,n) The only two subsets of faces $H_{r} \in C[G \backslash\{f\}]$ of the simple sets $G$ of (g,h) such that $H_{r} \cap \operatorname{Att}(G, F)=\emptyset$ (note that the third connected component of $G \backslash\{f\}$ in the case of $(\mathrm{g})$ does not verify the hypotheses of Proposition 31$)$. $(\ell, \mathrm{o})$ The respective closures $H=H_{r}^{-}$of the sets $H_{r}$ of $(\mathrm{k}, \mathrm{n})$ which verify the properties of Proposition 31. (m,p) The respective detachments of the sets $H$ of $(\ell, \mathrm{o})$ from $F$. As stated by Proposition 31 (iii), the complex $H$ of $(\ell)$ (resp. (o)) remains a simple set for the complex of (p) (resp. m). 


\subsection{1-D properties}

The following proposition states that a minimal simple set which is not a cell cannot contain any 1 -facets.

Proposition 37 Let $n \geq 1$. Let $F \leq \mathbb{F}^{n}$ be a cubical complex. Let $G \sqsubset F$ be a minimal simple set for $F$ such that $G$ is not a cell. Let $f \in G^{+}$be a facet of $G$. Then, $\operatorname{dim}(f) \geq 2$.

Proof Let us suppose that $\operatorname{dim}(f)<2$. From Proposition 35, we then have $\operatorname{dim}(f)=$ 1. Let $g_{1}, g_{2} \in \hat{f}$ be the two 0 -faces of $\hat{f}$. As $\hat{f}$ is not a simple cell for $F$, we have $g_{i} \in G, \operatorname{star}^{*}\left(g_{i}, F\right)$ is not connected, and $\{f\} \in C\left[\operatorname{star}^{*}\left(g_{i}, F\right)\right] \cap C\left[\operatorname{star}^{*}\left(g_{i}, G\right)\right]$ for $i \in[1,2]$, in contradiction with Proposition 33. Then, $\operatorname{dim}(f)>1$.

The following proposition, which states that a minimal simple set of dimension 1 is necessarily a simple cell, is a direct consequence of Proposition 37.

Proposition 38 Let $n \geq 1$. Let $F \leq \mathbb{F}^{n}$ be a cubical complex. Let $G \sqsubset F$ be a 1-D minimal simple set for $F$. Then $G$ is a simple cell for $F$.

The following proposition can easily be proved by induction from Propositions 30 and 31 .

Proposition 39 Let $n \geq 1$. Let $F \leq \mathbb{F}^{n}$ be a cubical complex. Let $G \sqsubset F$ be a simple set for $F$ such that $G$ is not a cell and $G^{+}$contains at least a 1-facet. Then:

(i) $\exists H \sqsubset G$ such that $\operatorname{Att}(H, F)$ is a 0 -cell;

(ii) $\forall H \sqsubset G$ such that $\operatorname{Att}(H, F)$ is a 0 -cell:

(a) $H$ is a simple set for $F$

(b) $G \otimes H$ is a simple set for $F \otimes H$.

Remark 40 Note that, based on this proposition, we can also prove by induction that a simple set can be detached (in a non-deterministic fashion) by iterative removal of simple 1-cells and/or (not necessarily minimal) simple sets composed of facets of dimension $\geq 2$. From an algorithmic point of view, this result implies that it is sufficient to only detect the simple 1-cells and the simple sets which do not contain 1-facets. Such simple sets are necessarily located in the connected components of complexes from which all the principal 1-cells have been detached.

The following proposition is a direct consequence of Propositions 38 and 39.

Proposition 41 Let $n \geq 1$. Let $F \leq \mathbb{F}^{n}$ be a cubical complex. Let $G \sqsubset F$ be a simple set for $F$ such that $\operatorname{dim}(G)=1$. If $G$ is not a cell, then:

(i) $\exists H \sqsubset G$ such that $H$ is a simple cell for $F$;

(ii) $\forall H \sqsubset G$ such that $H$ is a simple cell for $F, G \otimes H$ is a simple set for $F \otimes H$. 


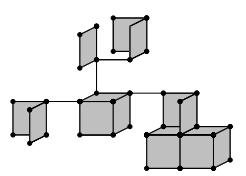

(a)

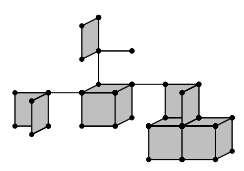

(d)

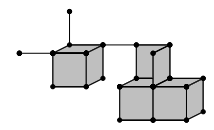

(g)

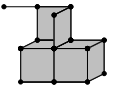

(j)

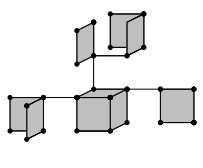

(b)

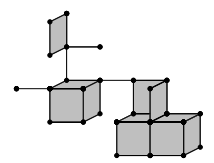

(e)

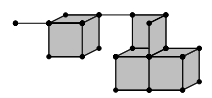

(h)

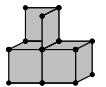

$(\mathrm{k})$

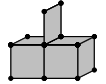

(c)

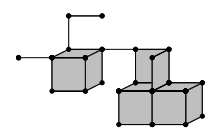

(f)

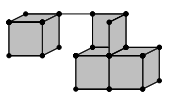

(i)

Fig. 23. (a) A complex $F$. (b) A simple set $G \sqsubset F$ of $F$. (c) The detachment of $G$ from $F$. (d- $\ell$ ) Successive detachments, from $F$, of simple 1-cells and simple sets composed of facets of dimension $\geq 2$, obtained from $G$ (see Proposition 39 and Remark 40).

Remark 42 The results of Proposition 41 cannot be extended to simple sets $G$ such that $\operatorname{dim}(G)=3$ (and a fortiori $\operatorname{dim}(G)>3$ ), the Bing's house providing an obvious counter-example. The extension of this proposition to $\operatorname{dim}(G)=2$ (which actually depends on the dimension of $\mathbb{F}^{n}$ ) will be considered in further works (see next section).

\section{Discussion and perspectives}

Simple sets are objects which, to our knowledge, have never been considered and, a fortiori, studied. From an algorithmic point of view, it is important to enable their detection (if possible with the lowest computational cost) and their removal (by direct or "step by step" strategies) to design efficient topology-preserving reduction procedures.

The propositions enunciated in Sections 5 and 6 lead to the following (non-exhaustive) list of considerations, which are valid independently of the dimension of the complex $F$ to be reduced nor the dimension of the space $\mathbb{F}^{n}$ in which it lies:

- any simple set $G$ of $F$ admits a subcomplex $H$ which is principal for $F$, and whose 
detachment from $F$ has the same effect as the detachment of $G$ (Proposition 19);

- any simple set $G$ of $F$ can be fully detached from $F$ by iterative (or parallel, since the order does not matter) detachment of its principal subcomplexes "sharing" their attachment with $G$ (Proposition 24), and a fortiori by iterative or parallel detachment of its connected components (Proposition 25);

- any simple set $G$ of $F$ can be fully detached from a complex by iterative (and non-deterministic) detachment of its principal subcomplexes attached to $F$ by a 0-face (Proposition 31), and in particular simple 1-cells and simple sets not containing 1-facets (Proposition 39);

- any simple set $G$ of $F$ of dimension 1 can be fully detached from $F$ by iterative (and non-deterministic) detachment of simple 1-cells composing it (Proposition 41).

In particular, it is then sufficient to detect principal, connected simple sets, without "isthmuses" (i.e. without 0-faces verifying the hypotheses of Propositions 30 and 31 ) to completely deal with the issue of removing simple sets. These properties can be used to limit the study of simple sets to a much smaller family whose knowledge is sufficient to lead to topology-preserving simple set-based reduction procedures guarantying the removal of all simple sets from a complex.

The removal of simple sets of dimension 1 can be handled by only considering simple 1-cells. The next step of this work will now consist in studying the case of dimension 2, first in "general" 2-D spaces (i.e. pseudo-manifolds), and then in more classical spaces of higher dimension (i.e. $\mathbb{F}^{n}$, with $n \geq 3$ ), to provide characterisations enabling the development of reduction procedures guarantying the complete detachment of all 2-D simple sets. In this context, it will be shown that the notion of minimal simple set (equivalent to the notion of simple cell at dimension 1, property which will no longer be necessarily true in higher dimensions) and the various properties introduced and studied in this paper, will be of precious use (see e.g. Passat et al. (2009); Mazo and Passat (2009)).

\section{Acknowledgements}

We warmly thank Michel Couprie and Gilles Bertrand for their important comments and suggestions.

We are grateful to the anonymous reviewers for their constructive comments, which led to significant improvement of the quality of this article. 


\section{References}

Bertrand, G., 1994. Simple points, topological numbers and geodesic neighborhoods in cubic grids. Pattern Recognition Letters 15 (10), 1003-1011.

Bertrand, G., 1995. On P-simple points. Comptes Rendus de l'Académie des Sciences, Série Math. I (321), 1077-1084.

Bertrand, G., 2007. On critical kernels. Comptes Rendus de l'Académie des Sciences, Série Math. I (345), 363-367.

Bertrand, G., Couprie, M., 2008. Two-dimensional thinning algorithms based on critical kernels. Journal of Mathematical Imaging and Vision 31 (1), 35-56.

Bing, R., 1964. Some aspects of the topology of 3-manifolds related to the Poincaré conjecture. Lectures on Modern Mathematics II, 93-128.

Couprie, M., Bertrand, G., 2009. New characterizations of simple points in 2D, 3D and 4D discrete spaces. IEEE Transactions on Pattern Analysis and Machine Intelligence 31 (4), 637-648.

Couprie, M., Coeurjolly, D., Zrour, R., 2007. Discrete bisector function and Euclidean skeleton in 2D and 3D. Image and Vision Computing 25 (10), 15431556.

Davies, E., Plummer, A., 1981. Thinning algorithms: a critique and a new methodology. Pattern Recognition 14 (1-6), 53-63.

Dokládal, P., Lohou, C., Perroton, L., Bertrand, G., 1999. Liver blood vessels extraction by a 3-D topological approach. In: Taylor, C., Colchester, A. (Eds.), Medical Image Computing and Computer-Assisted Intervention - MICCAI'99, 2nd International Conference, Proceedings. Vol. 1679 of Lecture Notes in Computer Science. Springer, pp. 98-105.

Fourey, S., Malgouyres, R., 2003. A concise characterization of 3D simple points. Discrete Applied Mathematics 125 (1), 59-80.

Gau, C.-J., Kong, T., 2003. Minimal non-simple sets in 4D binary pictures. Graphical Models 65 (1-3), 112-130.

Giblin, P., 1981. Graphs, surfaces and homology. Chapman and Hall.

Kaczynski, T., Mischaikow, K., Mrozek, M., 2004. Computational Homology. Vol. 157 of Applied Mathematical Sciences. Springer.

Kong, T., Litherland, R., Rosenfeld, A., 1990. Problems in the topology of binary digital images. In: van Mill, J., Reed, G. (Eds.), Open Problems in Topology. Elsevier Science Publishers B.V. (North-Holland), Ch. 23, pp. 377-385.

Kong, T. Y., 1995. On topology preservation in 2-D and 3-D thinning. International Journal on Pattern Recognition and Artificial Intelligence 9 (5), 813-844.

Kong, T. Y., 1997. Topology-preserving deletion of 1's from 2-, 3- and 4dimensional binary images. In: Ahronovitz, E., Fiorio, C. (Eds.), Discrete Geometry for Computer Imagery - DGCI'97, 7th International Workshop, Proceedings. Vol. 1347 of Lecture Notes in Computer Science. Springer, pp. 3-18.

Kong, T. Y., Rosenfeld, A., 1989. Digital topology: introduction and survey. Computer Vision, Graphics and Image Processing 48 (3), 357-393.

Kovalevsky, V., 1989. Finite topology as applied to image analysis. Computer Vision, Graphics and Image Processing 46 (2), 141-161. 
Ma, C., 1994. On topology preservation in 3D thinning. Computer Vision, Graphics and Image Processing 59 (3), 328-339.

Mazo, L., Passat, N., 2009. 2-D simple sets in n-D cubic grids. Discrete \& Computational Geometry (In Press).

Morgenthaler, D., 1981. Three-dimensional simple points: serial erosion, parallel thinning, and skeletonization. Tech. Rep. TR-1005, University of Maryland.

Passat, N., Couprie, M., Bertrand, G., 2007. Topological monsters in $\mathbb{Z}^{3}$ : A nonexhaustive bestiary. In: Bannon, G., Barrera, J., Braga-Neto, U., Hirata, N. (Eds.), International Symposium on Mathematical Morphology - ISMM'07, 8th International Symposium, Proceedings. Vol. 2. INPE, pp. 11-12.

Passat, N., Couprie, M., Bertrand, G., 2008. Minimal simple pairs in the 3-D cubic grid. Journal of Mathematical Imaging and Vision 32 (3), 239-249.

Passat, N., Couprie, M., Mazo, L., Bertrand, G., 2009. Topology-preserving thinning in 2-D pseudomanifolds. In: Brlek S., Provençal X., Reutenauer C. (Eds.), Discrete Geometry for Computer Imagery - DGCI'09, 15th International Conference, Proceedings (In Press).

Passat, N., Ronse, C., Baruthio, J., Armspach, J.-P., Bosc, M., Foucher, J., 2005. Using multimodal MR data for segmentation and topology recovery of the cerebral superficial venous tree. In: Bebis, G., Boyle, R., Koracin, D., Parvin, B. (Eds.), International Symposium on Visual Computing - ISVC'05, 1st International Symposium, Proceedings. Vol. 3804 of Lecture Notes in Computer Science. Springer, pp. 60-67.

Ronse, C., 1988. Minimal test patterns for connectivity preservation in parallel thinning algorithms for binary digital images. Discrete Applied Mathematics 21 (1), 67-79.

Rosenfeld, A., 1970. Connectivity in digital pictures. Journal of the Association for Computer Machinery 17 (1), 146-160.

Whitehead, J. H. C., 1938. Simplicial spaces, nuclei and $m$-groups. Proceedings of the London Mathematical Society s2-45 (1), 243-327. 
Supplementary material for on-line publication only Click here to download Supplementary material for on-line publication only: isorot.sty

\section{ty}

.

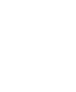
. (1) . . . .
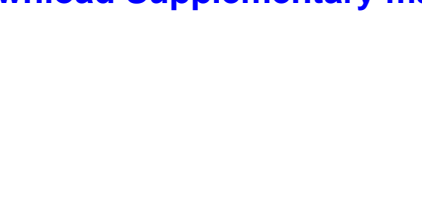